\title{
Rising trends and persisting inequalities in cesarean section rates in Nepal: evidence from demographic and health surveys 2006-2016
}

Kiran Acharya ( $\square$ acharya.kiran1@gmail.com )

New ERA https://orcid.org/0000-0002-7757-0066

Yuba Raj Paudel

Alberta Health Services

Chandra Mani Dhungana

United Nations Population Fund

Research article

Keywords: cesarean section, childbirth, over-use, inequality, Nepal

Posted Date: April 22nd, 2020

DOI: https://doi.org/10.21203/rs.3.rs-15560/v2

License: (우 (i) This work is licensed under a Creative Commons Attribution 4.0 International License. Read Full License 


\section{Abstract}

Background Sustainable development goals require member countries to reduce maternal mortality ratio below 70 per 100,000 live births by 2030 . Addressing inequalities in accessing emergency obstetric care is crucial for reducing the maternal mortality ratio. This study was undertaken to examine the time trends and sociodemographic inequalities in the utilization of cesarean section (CS) in Nepal during the period of 2006 and 2016.

Methods Data from the Nepal Demographic and Health Surveys (NDHS) 2006, 2011 and2016 were sourced for this study. Women who had a live birth in the last five years of the survey (most recent birth if there were two or more childbirths) were the unit of analysis for this study. Absolute and relative inequalities in CS rates were expressed in-terms of rate difference and rate ratios, respectively. We used binary logistic regression models to assess the rate of cesarean sections by background socio-demographic characteristics of women.

Results Age and parity adjusted CS rates were found to have increased almost three-fold (from $3.2 \%, 95 \%$ Cl:2.14.3 in 2006 to $10.5 \% ; 95 \%$ Cl:8.9-11.9 in 2016) over the decade. In 2016, women from Mountain region (3.0\%;95\% Cl:1.1-4.9), those from poorest wealth quintile $(2.4 \%, 95 \% \mathrm{Cl}:(1.2-3.7)$ and those living in province $6(2.4 \%, 95 \%$ $\mathrm{Cl} 1$ 1.3-3.5) had CS rate below $5 \%$. Whereas, women from the richest income quintile (25.1\%,95\% Cl:20.2-30.1), with higher education $(21.2 \%, 95 \% \mathrm{Cl}: 14.7-27.8)$ and those delivering in private facilities (37.1\%,95\% Cl:30.5-43.7) had CS rate above $15 \%$. Women from the richest income quintile (OR-3.3,95\% Cl: $1.6-7.0)$ and those delivered in private/NGO-run facilities (OR-3.6;95\% Cl:2.7-4.9) were more than three times more likely to deliver by CS compared to women from the poorest income quintile and those delivering in public facilities, respectively.

Conclusion To improve maternal and newborn health, strategies need to be revised to address the underuse of Csection in poor, mountain region and province 2, province 5 , province 6 and province 7 so that universal access to comprehensive sexual and reproductive health care services is ensured. Simultaneously, there is a pressing need for policies, guidelines and continuous monitoring of CS rates to reduce overuse in rich women, women with higher education and those delivered in private facilities.

\section{Background}

Caesarean section(CS) is one of the vital interventions to save lives of mothers and babies at the time of life threatening complications during pregnancy and child birth(1). There is a growing public health concern that population-based CS rates in some countries have reached above the WHO recommended level $(2,3)$. Nonmedical factors such as monetary incentives for health care providers and patient preferences are suggested to be key factors for unnecessary CS(4).

Government of $\mathrm{Nepal}(\mathrm{GoN})$ aims to reduce maternal and child mortality at the critical time of childbirth by ensuring basic emergency obstetric and newborn care and Comprehensive emergency obstetric and newborn care services to all women who need it(5). Nepal Health Sector strategy has highlighted equitable access to health services and leaving no one behind as one of the major foci of health sector (6). Additionally, in line with sustainable development goals, GoN strives to reduce Maternal mortality ratio to less than 70 per 100,000 live births and to reduce newborns and children deaths due to preventable causes to below one per cent(7). It is critical to have an easy access to emergency obstetric and newborn care to achieve these goals. 
Nepal's Safe Motherhood Program has been operational since 1997 with the aim of reducing maternal and newborn mortality. Nepal introduced maternity incentive scheme in 2005 (popularly known as Aama Program) to reduce financial barriers to reach health facility for institutional delivery. Additionally, GoN has rolled out free delivery care including CS nationwide by abolishing all user fees since 2009 from accredited facilities(8). Furthermore, current Aama Program provides a cash incentive of NPR 3,000 (USD 30 approximately) in mountain districts, NPR 2,000 (USD 20 approximately) in Hill districts, and NPR 1,000 (USD 10 approximately) in Terai districts to women delivering at health institutions(9). This also includes NPR 800 (USD 8 approximately) for women who completed four antenatal care as per GoN protocol. Health facilities also receive a case-based payment for providing free delivery according to type of delivery (normal deliveries: USD 10 for HFs below 25 beds, USD 15 for HFs above 25 beds, complicated deliveries: USD 30 and CS: USD 70)(10-12). Moreover, GoN has established an emergency referral fund in order to airlift women from geographically remote regions to referral centers in case of complications(5). As a result of these demand-side and supply-side policies, institutional deliveries in Nepal have considerably increased from $9 \%$ in 1996(13) to 57\% in 2016(14). However, given the geographical diversity and socio-cultural barriers, reaching poor, marginalized, uneducated and women living in the remote and rural areas is still a big concern $(15,16)$. Furthermore, Mehata et al showed significant inequalities in CS in Nepal by place of residence, wealth quintile, age of the mother, educational status and caste/ethnicity (17).

CS are not easily accessible to women from marginalized sections of the communities and those living in remote areas even when there is a strong medical indication (18). Simultaneously, unnecessary use is common in private hospitals(3), urban areas(19) and among higher educated mothers(20). Although increasing number of private and community hospitals are now implementing Aama program, charging fees for CS is common (21). In resource-poor settings such as Nepal, poor quality of obstetric care (lack of support and monitoring of child birth process, lack of pain management) resulting in less confidence to health care is thought to have increased demand for CS among upper and middle class women $(22,23)$. Since, private hospitals in Nepal are hitherto largely unregulated, high CS rate in private hospitals may be the result of 'on-demand' or 'provider-initiated' for monetary incentives $(16,24)$.

Although both underuse and overuse were associated with poor health outcomes for mothers and newborn, determining optimal CS rate at the population level is a herculean task(25). WHO's new statement released in 2015 recommended that the CS rates should be below 10\%(25). Besides poor health outcomes, CS is associated with high cost to the health care system and to the families(26). Furthermore, as in other settings(27), surge in CS rate has received increasing attention in Nepal on the grounds of women's rights, over-medicalization of birth and abuse of technology(24). Therefore, it is imperative to have an optimal CS rate at a population level while protecting the rights of women to have CS when there is a medical indication. Hence, it is important to monitor service utilization pattern and inequalities by population groups to assess program and policy outcomes. Therefore, by utilizing the most recent household level data from last three rounds of DHS surveys we present the trend and social determinants of inequalities in utilization of CS in Nepal.

\section{Methods}

\section{Data Source}


We used data from three rounds of the Nepal Demographic and Health Survey (NDHS) conducted in 2006, 2011 and 2016. New ERA, a local research firm in Nepal was responsible in implementing all these surveys under the leadership of Ministry of Health and population (MoHP) and technical assistance from ICF. Briefly, NDHS collected nationally representative data every 5 years on a broad range of issues including fertility, reproductive and maternal health, nutrition, and child health. In each round, a nationally representative sample of households was obtained using stratified cluster sampling approach. NDHS 2006 and 2011 used two-stage stratified cluster sampling to select households. Stratification was done by urban/rural place of residence. In the first stage, Primary Sampling Units (PSUs) were selected using probability proportional to size. In the second stage, households were selected using systematic sampling from individual PSU in rural areas. However, three-stage stratified cluster sampling technique was used for selecting households in urban areas for NDHS 2016 i.e in the first stage, PSUs were chosen by probability proportional to size followed by random selection of enumeration areas (EA) from PSUs in second stage and in third stage, households were selected systematically from selected EAs. Details of the survey design and data collection procedure is available in respective survey reports(14, 28, 29). Women aged 15-49 years who had a live-birth within 5 years preceding the survey comprised the unit of analysis for the current study. In the case when there was more than single birth within five years' period, we considered the most recent birth in our study.

\section{Measures}

The dependent variable for this study was whether the last live birth of a woman aged 15-49 years was conducted by C-section. The DHS surveys used same questions: "Was the (name of the last child) delivered by caesarian section?' The responses were recorded as a binary variable either 1 meaning 'Yes' or 0 meaning 'No.'

Based on the available literature(30-32), the independent variables selected for this study include: women's age categories, parity(2 or less, 3-4, more than 4), women's education, women's current working status(Currently working, not working), wealth index, ecological region(Mountain, Hill, Terai), province(Province 1-7), place of residence(urban/rural), ethnicity and place of delivery. The wealth index used for this study were calculated by principal component analysis based on the easy-to-collect data on a household's ownership of selected assets, such as televisions and bicycles; materials used for housing construction; and access to water and sanitation facilities. Households were then categorized to the poorest (Q1), poorer, middle, richer, or the richest (Q5) group $(30,33)$.

\section{Data Analysis}

Initially, we computed crude CS rates by women's socio-demographic characteristics. Then, we computed the adjusted CS rates and their $95 \%$ confidence intervals standardizing for age and parity using direct standardization method using the sample of NDHS 2016 as the standard population. We then created the standard weight of age and parity separately and combined them for each NDHS. Further, we adjusted them for each covariate using command "stdize" and "stdweight". To measure the inequalities, two inequality indicators-the ratio between the highest rank and the lowest rank (ratio of Q1 to Q5 for health indicators and the ratio of Q5 to Q1 for indicators of health care) $(34,35)$ and, Rich Poor difference were calculated for this study. The Rate ratio indicator links the level of health or use of health services between the highest and lowest rank. Rate difference provides an absolute difference in prevalence of caesarian section between the highest and lowest rank. To some extent, 
these two indicators i.e absolute inequality in terms of rate difference (Q5-Q1) and relative inequality in terms of rate ratio(Q5/Q1) was done for each of the survey periods(35-38). Presenting both relative and absolute measures of inequalities is important for increasing transparency, and to provide unbiased evidence for policy making (39) however these two frequently used measures of inequality are easy to understand, but comparisons are limited to two extreme groups rather than covering the full population variety $(35,40)$. We conducted binary logistic regression analysis adjusting for women's age and parity to estimate the odd ratios of CS according to the women's characteristics. All independent variables were tested for collinearity before running logistic regression. Since no collinearity was detected, all variables were included in the regression model. The level of statistical significance was set to 0.05 . Although recent guideline has suggested CS rate of $10 \%$ as adequate at population level(25), we used $5 \%$ as minimum rate and $15 \%$ as maximum rate to interpret underuse and overuse of CS based on previously accepted standards $(41,42)$. We used sampling weights to adjust for variations in the selection probabilities and interviews among respondents and "svyset" command was used to account for complex survey design and to provide unbiased estimate. All analyses were performed using STATA version 15.0.

\section{Ethical Considerations}

Nepal DHS surveys were reviewed and approved by Nepal Health Research Council (NHRC) and the Institutional Review Board of ICF. The interviewers pursued informed consent from the women before the interviews as per the guideline of NHRC. We used de-identified publicly available data upon the request from DHS program (https://dhsprogram.com/Data/terms-of-use.cfm). This is the secondary analysis and the publicly available datasets did not include individual identifiers and thus did not require ethics approval.

\section{Results}

The analysis revealed most of the women were 20-29 years old and constituted about two- third of the total population in all three surveys. Proportion of women with higher education increased remarkably from $6.2 \%$ in 2006 to $21.4 \%$ in 2016 . Inverse to higher education, the proportion of working women decreased from $69.0 \%$ in the 2006 to $49.4 \%$ in the 2016. Concurrently, we found little difference in the distribution of women's wealth index. Among the respondents, less than $20 \%$ belonged to the rich and richest group in all three surveys. During 2006 to 2011 more than $80 \%$ of the women lived in rural areas while it decreased to $46.0 \%$ in 2016 . About one third of the women belonged to Newar/janjaties in all surveys. The proportion of women giving birth at health facilities (public and private sectors) increased rapidly over the decade. The proportion women giving birth at public facility increased by more than three folds, from $13.1 \%$ in 2006 to $43.1 \%$ in 2016 . While proportion of birth in private facilities also doubled during the same period from $4.6 \%$ to $10.8 \%$ (Table 1 ). 
Table 1.Socio-demographic characteristics and the use of cesarean section from the Nepal Demographic and Health Surveys (NDHS) in 2006, 2011, and 2016

\begin{tabular}{|c|c|c|c|c|c|c|}
\hline \multirow[t]{3}{*}{ Characteristics } & \multirow{2}{*}{\multicolumn{2}{|c|}{$\begin{array}{c}\text { NDHS } 2006 \\
\mathrm{~N}=5545)\end{array}$}} & \multirow{2}{*}{\multicolumn{2}{|c|}{$\begin{array}{c}\text { NDHS } 2011 \\
(\mathrm{~N}=5391)\end{array}$}} & \multicolumn{2}{|c|}{ NDHS 2016} \\
\hline & & & & & \multicolumn{2}{|c|}{$(\mathrm{N}=5060)$} \\
\hline & Frequency & $\%$ & Frequency & $\%$ & Frequency & $\%$ \\
\hline \multicolumn{7}{|l|}{ Maternal age, (years) } \\
\hline $15-19$ & 368 & 6.6 & 381 & 7.1 & 391 & 7.7 \\
\hline $20-24$ & 1,931 & 34.8 & 1,802 & 33.4 & 1,665 & 32.9 \\
\hline $25-29$ & 1,742 & 31.4 & 1,736 & 32.2 & 1,780 & 35.2 \\
\hline $30-34$ & 785 & 14.2 & 845 & 15.7 & 806 & 15.9 \\
\hline $35-39$ & 465 & 8.4 & 402 & 7.5 & 297 & 5.9 \\
\hline $40-44$ & 196 & 3.5 & 169 & 3.1 & 97 & 1.9 \\
\hline $45-49$ & 58 & 1.0 & 57 & 1.1 & 24 & 0.5 \\
\hline \multicolumn{7}{|l|}{ Parity } \\
\hline 2 or less & 3,132 & 56.5 & 3,293 & 61.1 & 3,472 & 68.6 \\
\hline $3-4$ & 1,459 & 26.3 & 1,405 & 26.1 & 1,161 & 22.9 \\
\hline More than 4 & 954 & 17.2 & 693 & 12.9 & 427 & 8.4 \\
\hline \multicolumn{7}{|l|}{ Education } \\
\hline No education & 3,343 & 60.3 & 2,550 & 47.3 & 1,733 & 34.3 \\
\hline Primary & 1,009 & 18.2 & 1,079 & 20.0 & 1,019 & 20.1 \\
\hline some secondary & 848 & 15.3 & 1,039 & 19.3 & 1,226 & 24.2 \\
\hline SLC and above ${ }^{1}$ & 345 & 6.2 & 723 & 13.4 & 1,082 & 21.4 \\
\hline \multicolumn{7}{|l|}{ Current working status } \\
\hline Working & 3,824 & 69.0 & 2,961 & 54.9 & 2,498 & 49.4 \\
\hline Not working & 1,721 & 31.0 & 2,431 & 45.1 & 2,562 & 50.6 \\
\hline \multicolumn{7}{|l|}{ Wealth index } \\
\hline Poorest & 1,412 & 25.5 & 1,390 & 25.8 & 1,082 & 21.4 \\
\hline Poorer & 1,180 & 21.3 & 1,182 & 21.9 & 1,072 & 21.2 \\
\hline Middle & 1,132 & 20.4 & 1,133 & 21.0 & 1,121 & 22.2 \\
\hline Richer & 983 & 17.7 & 938 & 17.4 & 1,036 & 20.5 \\
\hline Richest & 838 & 15.1 & 748 & 13.9 & 748 & 14.8 \\
\hline \multicolumn{7}{|l|}{ Residence } \\
\hline Urban & 677 & 12.2 & 503 & 9.3 & 2,730 & 54.0 \\
\hline Rural & 4,868 & 87.8 & 4,888 & 90.7 & 2,330 & 46.0 \\
\hline \multicolumn{7}{|l|}{ Ecological region } \\
\hline Mountain & 483 & 8.7 & 428 & 7.9 & 361 & 7.1 \\
\hline Hill & 2,261 & 40.8 & 2,130 & 39.5 & 1,911 & 37.8 \\
\hline Terai & 2,802 & 50.5 & 2,833 & 52.6 & 2,789 & 55.1 \\
\hline \multicolumn{7}{|l|}{ Province } \\
\hline Province1 & 920 & 16.6 & 1,135 & 21.0 & 819 & 16.2 \\
\hline Province2 & 1,171 & 21.1 & 1,146 & 21.3 & 1,367 & 27.0 \\
\hline Province3 & 920 & 16.6 & 705 & 13.1 & 813 & 16.1 \\
\hline Province4 & 571 & 10.3 & 589 & 10.9 & 388 & 7.7 \\
\hline Province5 & 822 & 14.8 & 810 & 15.0 & 899 & 17.8 \\
\hline Province6 & 341 & 6.1 & 401 & 7.4 & 338 & 6.7 \\
\hline Province7 & 800 & 14.4 & 605 & 11.2 & 437 & 8.6 \\
\hline \multicolumn{7}{|l|}{ Caste/Ethnicity } \\
\hline Brahamin/Chettri & 1,639 & 29.6 & 1,618 & 30.0 & 1,396 & 27.6 \\
\hline Terai/Madhesi other & 685 & 12.4 & 558 & 10.4 & 1,021 & 20.2 \\
\hline Dalits & 884 & 15.9 & 959 & 17.8 & 695 & 13.7 \\
\hline Newar/Janajatis & 1,934 & 34.9 & 1,892 & 35.1 & 1,573 & 31.1 \\
\hline Muslim/Other & 402 & 7.3 & 364 & 6.7 & 375 & 7.4 \\
\hline \multicolumn{7}{|l|}{ Place of delivery } \\
\hline Public facility & 724 & 13.1 & 1,399 & 26.0 & 2,183 & 43.1 \\
\hline Private facility/NGOs & 256 & 4.6 & 506 & 9.4 & 548 & 10.8 \\
\hline
\end{tabular}




\begin{tabular}{|l|r|r|r|r|r|r|} 
Outside Nepal & NA & NA & NA & NA & 171 & 3.4 \\
\hline Home & 4,492 & 81.0 & 3,402 & 63.1 & 2,096 & 41.4 \\
\hline Others & 73 & 1.3 & 85 & 1.6 & 61 & 1.2 \\
\hline Cesarean section & & & & & & \\
\hline Yes & 148 & 2.7 & 248 & 4.6 & 457 & 9.0 \\
\hline No & 5,397 & 97.3 & 5,143 & 95.4 & 4,603 & 91 \\
\hline
\end{tabular}

Cesarean section was determined for the most recent birth of women aged 15-49 years who had a livebirth five years preceding the surveys.

All percentages are weighted, so the absolute number of participants doesn't perfectly correspond to percentages. SLC stands for School Leaving certificate and SLC and above means higher education

NA: not available

Crude C-section increased from 2.7\% in 2006 to 9.0\% in 2016, with large and increasing absolute disparities in Csection according to women's socio-demographic characteristics (Table 2). A higher education level was associated with a markedly higher C-section rate in all three surveys, specifically; in 2016 this rate was at $19.5 \%$. Furthermore, higher C-section rates $(28.2 \%$ in 2016) was observed among the women from the richest income quintile and the lowest rate (2.4\%) among women from the poorest income quintile. Provincial analysis showed, province 3 had persistently higher C-section rate (6.0\%, 8.3\% and 17.4\% in 2006, 2011 and in 2016 respectively) followed by province 4 (1.7\%, 4.0\% and 16.7\% in 2006, 2011 and 2016 respectively). Similarly, women living in urban areas had higher $\mathrm{C}$-section rates than women did in rural areas, although the absolute difference reduced during 2016 compared with 2006 and 2011. Greater difference was seen in the utilization of C-section between public and private health facilities. This was true for all three rounds of the NDHS. The C-section rates in the public and private sectors were $13.9 \%$ and $18.4 \%$, respectively, in $2006,8.6 \%$ and $25.4 \%$, respectively, in 2011 , and $12.1 \%$ and $34.8 \%$, respectively, in 2016 . Among the different caste/ethnic groups, the Brahamin/Chettri had a higher C-section rate with an increase from 4.4\% in 2006 to 11.3\% in 2016 followed by Newar / Janajatis from $2.3 \%$ in 2006 to $10.2 \%$ in 2016 . There was an increasing trend of C-section among working and non-working women.

It is interesting to note that the proportion of CS among those delivered in HFs increased slightly from $15.1 \%$ in 2006 to $16.7 \%$ in 2016 (Table 2). Of the total deliveries at public facilities, those conducted by CS remained almost constant (12.3\% in 2006, and $12.6 \%$ in 2016). Meanwhile, among the total deliveries conducted in private facilities there was a sharp rise in CS from $15.8 \%$ in 2006 to $37.1 \%$ in 2016 (Table 3). 
Table 2.Crude cesarean section(CS) rates (\%) by socio-demographic characteristics from the Nepal Demographic and Health Surveys (NDHS) in 2006, 2011, and 2016

\begin{tabular}{|c|c|c|c|c|c|c|c|c|c|}
\hline \multirow[t]{2}{*}{ Characteristics } & \multicolumn{3}{|c|}{ NDHS 2006} & \multicolumn{3}{|c|}{ NDHS 2011} & \multicolumn{3}{|c|}{ NDHS 2016} \\
\hline & Total births & $\mathrm{CS}$ & $\%$ & Total births & $\mathrm{CS}$ & $\%$ & Total births & $\mathrm{CS}$ & $\%$ \\
\hline Total (N) & 5545 & 148 & 2.7 & 5391 & 248 & 4.6 & 5060 & 457 & 9 \\
\hline \multicolumn{10}{|l|}{ Maternal age, (years) } \\
\hline $15-19$ & 368 & 16 & 4.2 & 381 & 14 & 3.5 & 391 & 25 & 6.3 \\
\hline $20-24$ & 1,931 & 47 & 2.4 & 1,802 & 64 & 3.6 & 1,665 & 142 & 8.5 \\
\hline $25-29$ & 1,742 & 56 & 3.2 & 1,736 & 93 & 5.4 & 1,780 & 163 & 9.2 \\
\hline $30-34$ & 785 & 17 & 2.2 & 845 & 52 & 6.1 & 806 & 88 & 11 \\
\hline $35-39$ & 465 & 3 & 0.6 & 402 & 18 & 4.4 & 297 & 35 & 11.8 \\
\hline $40-44$ & 196 & 9 & 4.4 & 169 & 7 & 4.4 & 97 & 4 & 3.6 \\
\hline $45-49$ & 58 & 0 & 0 & 57 & $<1$ & 0.6 & 24 & 0 & 0 \\
\hline \multicolumn{10}{|l|}{ Parity } \\
\hline 2 or less & 3,132 & 129 & 4.1 & 3,293 & 203 & 6.2 & 3,472 & 407 & 11.7 \\
\hline $3-4$ & 1,459 & 7 & 0.5 & 1,405 & 43 & 3.0 & 1,161 & 42 & 3.6 \\
\hline More than 4 & 954 & 11 & 1.1 & 693 & 2 & 0.3 & 427 & 8 & 1.8 \\
\hline \multicolumn{10}{|l|}{ Education } \\
\hline No education & 3,343 & 37 & 1.1 & 2,550 & 45 & 1.8 & 1,733 & 78 & 4.5 \\
\hline Primary & 1,009 & 28 & 2.8 & 1,079 & 44 & 4.1 & 1,019 & 57 & 5.6 \\
\hline some secondary & 848 & 35 & 4.1 & 1,039 & 66 & 6.3 & 1,226 & 110 & 9 \\
\hline SLC and above & 345 & 48 & 13.9 & 723 & 93 & 12.9 & 1,082 & 211 & 19.5 \\
\hline \multicolumn{10}{|l|}{ Current working status } \\
\hline Working & 3,824 & 52 & 1.4 & 2,961 & 82 & 2.8 & 2,498 & 193 & 8 \\
\hline Not working & 1,721 & 96 & 5.6 & 2,431 & 166 & 6.8 & 2,562 & 264 & 10 \\
\hline \multicolumn{10}{|l|}{ Wealth index } \\
\hline Poorest & 1,412 & 11 & 0.8 & 1,390 & 14 & 1.0 & 1,082 & 26 & 2.4 \\
\hline Poorer & 1,180 & 6 & 0.5 & 1,182 & 10 & 0.8 & 1,072 & 46 & 4.2 \\
\hline Middle & 1,132 & 11 & 1.0 & 1,133 & 52 & 4.6 & 1,121 & 77 & 6.8 \\
\hline Richer & 983 & 19 & 2.0 & 938 & 67 & 7.1 & 1,036 & 97 & 9.4 \\
\hline Richest & 838 & 100 & 11.9 & 748 & 106 & 14.1 & 748 & 211 & 28.2 \\
\hline \multicolumn{10}{|l|}{ Residence } \\
\hline Urban & 677 & 57 & 8.4 & 503 & 77 & 15.3 & 2,730 & 320 & 11.7 \\
\hline Rural & 4,868 & 90 & 1.9 & 4,888 & 171 & 3.5 & 2,330 & 137 & 5.9 \\
\hline \multicolumn{10}{|l|}{ Ecological region } \\
\hline Mountain & 483 & 4 & 0.7 & 428 & 6 & 1.4 & 361 & 9 & 2.6 \\
\hline Hill & 2,261 & 70 & 3.1 & 2,130 & 78 & 3.7 & 1,911 & 214 & 11.2 \\
\hline Terai & 2,802 & 74 & 2.6 & 2,833 & 164 & 5.8 & 2,789 & 234 & 8.4 \\
\hline \multicolumn{10}{|l|}{ Province } \\
\hline Province1 & 920 & 19 & 2.1 & 1,135 & 74 & 6.5 & 819 & 104 & 12.7 \\
\hline Province2 & 1,171 & 25 & 2.1 & 1,146 & 47 & 4.1 & 1,367 & 69 & 5.0 \\
\hline Province3 & 920 & 55 & 6.0 & 705 & 59 & 8.3 & 813 & 141 & 17.4 \\
\hline Province4 & 571 & 10 & 1.7 & 589 & 24 & 4 & 388 & 65 & 16.7 \\
\hline Province5 & 822 & 27 & 3.3 & 810 & 30 & 3.7 & 899 & 57 & 6.4 \\
\hline Province6 & 341 & 5 & 1.4 & 401 & 4 & 1 & 338 & 7 & 2.2 \\
\hline Province7 & 800 & 7 & 0.8 & 605 & 11 & 1.8 & 437 & 13 & 3.1 \\
\hline \multicolumn{10}{|l|}{ Caste/Ethnicity } \\
\hline Brahamin/Chettri & 1,639 & 72 & 4.4 & 1,618 & 118 & 7.3 & 1,396 & 158 & 11.3 \\
\hline Terai/Madhesi other & 685 & 15 & 2.2 & 558 & 33 & 6 & 1,021 & 73 & 7.1 \\
\hline Dalits & 884 & 11 & 1.2 & 959 & 20 & 2.1 & 695 & 38 & 5.4 \\
\hline Newar/Janajatis & 1,934 & 45 & 2.3 & 1,892 & 64 & 3.4 & 1,573 & 160 & 10.2 \\
\hline Muslim/Other & 402 & 4 & 1.0 & 364 & 12 & 3.2 & 375 & 28 & 7.4 \\
\hline \multicolumn{10}{|l|}{ Place of delivery } \\
\hline Public facility & 724 & 101 & 13.9 & 1,399 & 120 & 8.6 & 2,183 & 265 & 12.1 \\
\hline Private facility/NGOs & 256 & 47 & 18.4 & 506 & 129 & 25.4 & 548 & 191 & 34.8 \\
\hline
\end{tabular}




\begin{tabular}{|l|r|r|r|r|r|r|r|r|r|} 
Outside Nepal & NA & NA & NA & NA & NA & NA & 171 & 1 & 0.8 \\
\hline Home & 4,492 & 0 & 0 & 3,402 & 0 & 0 & 2,096 & 0 & 0 \\
\hline Others & 73 & 0 & 0 & 85 & 0 & 0 & 61 & 0 & 0 \\
\hline
\end{tabular}

Cesarean section was determined for the most recent birth of women aged 15-49 years who had a live-birth five years preceding the surveys.

correspond to percentages.

All percentages are weighted, so the absolute number of participants doesn't perfectly

The question on C-section was asked only of women who delivered in a health facility while analysis was based on the number of total births.

NA: not available

Age- and parity-adjusted C-section rates increased from $3.2 \%$ in 2006 to $10.5 \%$ in 2016 representing almost a threefold increase during 10 years' period (Table 3). The greatest increase in CS was seen among women with a higher educational level, those in the richest wealth quintile and women who delivered in private facility. Similar patterns of socioeconomic differences were observed among women living in province 1, province 3 and province 4. The absolute inequality in CS rate by maternal educational status increased from $9.7 \%$ in 2006 to $15.2 \%$ in 2016. Similarly, the absolute inequality in CS rate by income quintiles more than doubled from $10.3 \%$ in 2006 to $22.7 \%$ in 2016. Furthermore, absolute inequality also increased for ecological region and provinces of Nepal. Notably, the absolute difference in CS rate between public and private facilities increased from $3.5 \%$ to $24.5 \%$.

Relative inequality among women decreased in most of the socio-demographic characteristics and geographical distribution. However, the relative inequality increased in the provinces (from 5.0 in 2006 to 7.3 in 2016) and by place of delivery (1.3 in 2006 to 2.9 in 2016). In 2016, the highest absolute inequalities were observed for place of delivery followed by wealth status, maternal education and province. The highest relative inequality was observed for wealth quintile, province, ecological region and maternal education. 
Table 3.Maternal age and Parity-adjusted cesarean section(CS) rates (\%) from the Nepal

Demographic and Health Surveys (NDHS) in 2006, 2011, and 2016

\begin{tabular}{|c|c|c|c|}
\hline Characteristics & NDHS 2006 & NDHS 2011 & NDHS 2016 \\
\hline Overall adjusted CS rates (\%) & $3.2(2.1-4.3)$ & $6.6(5.1-8.1)$ & $10.5(8.9-11.9)$ \\
\hline \multicolumn{4}{|l|}{ Education } \\
\hline No education & $1.3(0.5-2.1)$ & $4.2(2.7-5.7)$ & $6.3(3.5-9.0)$ \\
\hline Primary & $3.7(1.1-6.3)$ & $5.7(2.7-8.7)$ & $6.0(3.5-8.6)$ \\
\hline some secondary & $4.8(2.4-7.2)$ & $6.2(3.9-8.5)$ & $10.5(7.7-13.3)$ \\
\hline SLC and above & $11.0(7.8-14.2)$ & $17.4(11.3-23.5)$ & $21.2(14.7-27.8)$ \\
\hline Rate difference & 9.7 & 13.2 & 15.2 \\
\hline Rate Ratio & 8.5 & 4.1 & 3.5 \\
\hline \multicolumn{4}{|l|}{ Current working status } \\
\hline Working & $1.6(0.8-2.3)$ & $4.8(3.4-6.2)$ & $9.5(7.6-11.4)$ \\
\hline Not working & $6.6(4.2-9.0)$ & $8.7(6.6-10.7)$ & $11.6(9.4-13.9)$ \\
\hline Rate difference & 5.0 & 3.9 & 2.1 \\
\hline Rate Ratio & 4.1 & 1.8 & 1.2 \\
\hline \multicolumn{4}{|l|}{ Wealth index } \\
\hline Poorest & $1.4(0.1-2.6)$ & $2.7(0.8-4.6)$ & $2.4(1.2-3.7)$ \\
\hline Poorer & $0.5(0.03-0.9)$ & $1.3(0.1-2.6)$ & $5.2(3.0-7.4)$ \\
\hline Middle & $1.2(0.09-2.4)$ & $6.2(4.0-8.4)$ & $8.0(4.9-11.1)$ \\
\hline Richer & $1.4(0.6-2.3)$ & $8.7(5.7-11.6)$ & $9.3(6.7-12.0)$ \\
\hline Richest & $10.8(7.7-13.9)$ & $13.4(9.7-17.2)$ & $25.1(20.2-30.1)$ \\
\hline Rate difference & 10.3 & 12.1 & 22.7 \\
\hline Rate Ratio & 21.6 & 10.3 & 10.5 \\
\hline \multicolumn{4}{|l|}{ Residence } \\
\hline Urban & $8.0(5.7-10.4)$ & $14.8(10.8-18.8)$ & $12.4(10.4-14.4)$ \\
\hline Rural & $2.0(1.0-3.0)$ & $5.3(3.8-6.8)$ & $7.0(5.1-8.9)$ \\
\hline Rate difference & 6.0 & 9.5 & 5.4 \\
\hline Rate Ratio & 4.0 & 2.8 & 1.8 \\
\hline \multicolumn{4}{|l|}{ Ecological region } \\
\hline Mountain & $0.8(0.02-1.6)$ & $1.5(0.6-2.4)$ & $3.0(1.1-4.9)$ \\
\hline Hill & $3.6(2.0-5.2)$ & $6.7(4.9-8.5)$ & $12.2(9.9-14.5)$ \\
\hline Terai & $2.6(1.6-3.6)$ & $6.6(5.1-8.1)$ & $10.0(7.9-12.1)$ \\
\hline Rate difference & 2.8 & 5.2 & 9.2 \\
\hline Rate Ratio & 4.5 & 4.5 & 4.1 \\
\hline \multicolumn{4}{|l|}{ Province } \\
\hline Province1 & $1.9(0.6-3.1)$ & $6.7(4.0-9.4)$ & $12.2(8.8-15.6)$ \\
\hline Province 2 & $2.1(0.6-3.5)$ & $6.6(4.5-8.8)$ & $6.8(3.6-9.9)$ \\
\hline Province3 & $6.0(3.2-8.8)$ & $12.1(8.0-16.2)$ & $16.4(12.6-20.2)$ \\
\hline Province4 & $2.2(-0.05-4.5)$ & $4.9(2.3-7.6)$ & $17.5(12.5-22.4)$ \\
\hline Province5 & $3.3(1.6-5.1)$ & $3.9(2.1-5.7)$ & $7.8(5.0-10.5)$ \\
\hline Province6 & $3.0(-0.1-6.1)$ & $2.9(2.0-3.7)$ & $2.4(1.3-3.5)$ \\
\hline Province7 & $1.2(-0.1-2.6)$ & $2.8(1.4-4.3)$ & $5.7(2.7-8.7)$ \\
\hline Rate difference & 4.8 & 9.3 & 15.1 \\
\hline Rate Ratio & 5.0 & 4.3 & 7.3 \\
\hline
\end{tabular}




\begin{tabular}{|l|c|c|c|} 
Caste/Ethnicity & & & \\
\hline Brahamin/Chettri & $5.2(3.0-7.4)$ & $8.3(6.3-10.4)$ & $11.8(9.3-14.4)$ \\
\hline Terai/Madhesi other & $2.8(0.8-4.9)$ & $8.5(4.0-13.0)$ & $10.3(6.1-14.6)$ \\
\hline Dalits & $0.9(0.2-1.7)$ & $2.7(0.7-4.6)$ & $7.8(4.0-11.6)$ \\
\hline Newar/Janajatis & $2.9(1.0-4.9)$ & $5.2(3.3-7.1)$ & $10.2(7.8-12.6)$ \\
\hline Muslim/Other & $0.8(-0.2-1.8)$ & $4.5(0.5-8.6)$ & $11.1(4.1-18.2)$ \\
\hline Rate difference & 4.4 & 5.8 & 4.0 \\
\hline Rate Ratio & 6.5 & 3.1 & 1.5 \\
\hline Place of delivery* & & & $12.6(10.4-14.8)$ \\
\hline Public facility & $12.3(9.1-15.6)$ & $11.1(8.4-13.9)$ & $37.1(30.5-43.7)$ \\
\hline Private facility/NGOs & $15.8(9.9-21.6)$ & $26.4(20.8-31.9)$ & $0.7(-0.6-2.0)$ \\
\hline Outside Nepal & NA & NA & 24.5 \\
\hline Rate difference & 3.5 & 15.3 & 2.9 \\
\hline Rate Ratio & 1.3 & 2.4 & \\
\hline
\end{tabular}

*Home deliveries were excluded from the analysis.

Cesarean section was determined for the most recent birth of women aged 15-49 years who had a live-birth five years preceding the surveys. The study sample of NDHS 2016 ( $N=5060)$ was used as the standard population in the direct standardization.

Note: For each survey, categories with the highest and lowest CS rate were used to calculate rate difference and rate ratios.

NA: not available

Table 4 shows age and parity-adjusted odds ratios of C-section using binary logistic regression. The likelihood of C-section was three times higher among women belonging to the richest group in 2016 (OR 3.3,95\% Cl: 1.6-7.0) compared to the poorest income quintile. Similarly, women who delivered in private facilities were more than three times likely to have C-section in both 2011 (OR: 3.2,95\% Cl: 2.2-4.6) and 2016 NDHS (OR: 3.6,95\% Cl: 2.7-4.9) surveys compared to those delivered in public facilities. 
Table 4. Binary logistic regression analysis of cesarean section(CS) from the Nepal Demographic and Health Surveys (NDHS) in 2006, 2011, and 2016

\begin{tabular}{|c|c|c|c|}
\hline Characteristics & NDHS 2006 & NDHS 2011 & NDHS 2016 \\
\hline \multicolumn{4}{|l|}{ Education } \\
\hline No education & 1.00 (Reference) & 1.00 (Reference) & 1.00 (Reference) \\
\hline Primary & $1.0(0.5-2.1)$ & $1.6(0.8-3.1)$ & $0.7(0.4-1.1)$ \\
\hline some secondary & $0.5(0.2-1.1)$ & $1.5(0.8-3.0)$ & $0.6(0.3-1.1)$ \\
\hline SLC and above & $0.6(0.2-1.5)$ & $1.4(0.6-3.0)$ & $0.7(0.4-1.6)$ \\
\hline \multicolumn{4}{|l|}{ Current working status } \\
\hline Working & 1.00 (Reference) & 1.00 (Reference) & 1.00 (Reference) \\
\hline Not working & $2.1^{* *}(1.3-3.5)$ & $1.5(0.8-3.0)$ & $1.1(0.8-1.4)$ \\
\hline \multicolumn{4}{|l|}{ Wealth index } \\
\hline Poorest & 1.00 (Reference) & 1.00 (Reference) & 1.00 (Reference) \\
\hline Poorer & $0.2(0.0-1.1)$ & $0.3(0.1-1.2)$ & $1.0(0.5-1.9)$ \\
\hline Middle & $0.3(0.1-1.5)$ & $0.9(0.3-2.7)$ & $1.1(0.6-2.2)$ \\
\hline Richer & $0.3(0.1-1.8)$ & $0.6(0.2-1.8)$ & $1.1(0.6-2.0)$ \\
\hline Richest & $1.1(0.2-6.3)$ & $0.5(0.2-1.6)$ & $3.3 * *(1.6-7.0)$ \\
\hline \multicolumn{4}{|l|}{ Residence } \\
\hline Urban & 1.00 (Reference) & 1.00 (Reference) & 1.00 (Reference) \\
\hline Rural & $1.3(0.7-2.3)$ & $0.5^{* *}(0.3-0.8)$ & $1.2(0.9-1.6)$ \\
\hline \multicolumn{4}{|l|}{ Ecological region } \\
\hline Mountain & 1.00 (Reference) & 1.00 (Reference) & 1.00 (Reference) \\
\hline Hill & $0.9(0.2-4.4)$ & $1.0(0.5-1.9)$ & $1.5(0.7-3.2)$ \\
\hline Terai & $1.0(0.2-6.1)$ & $1.5(0.7-3.1)$ & $1.6(0.7-3.6)$ \\
\hline \multicolumn{4}{|l|}{ Province } \\
\hline Province1 & 1.00 (Reference) & 1.00 (Reference) & 1.00 (Reference) \\
\hline Province2 & $1.7(0.6-4.7)$ & $1.1(0.5-2.2)$ & $0.4^{* *}(0.2-0.8)$ \\
\hline Province3 & $1.2(0.5-3.2)$ & $1.9 *(1.1-3.4)$ & $1.1(0.7-1.9$ \\
\hline Province4 & $0.7(0.1-3.9)$ & $1.0(0.5-1.8)$ & $1.7 *(1.1-2.8)$ \\
\hline Province5 & $1.7(0.7-4.2)$ & $0.8(0.4-1.6)$ & $0.4^{* * *}(0.3-0.7)$ \\
\hline Province6 & $0.7(0.2-3.0)$ & $0.7(0.2-2.8)$ & $0.5(0.2-1.1)$ \\
\hline Province7 & $0.7(0.2-2.5)$ & $0.5(0.3-1.2)$ & $0.4^{* *}(0.2-0.7)$ \\
\hline \multicolumn{4}{|l|}{ Caste/Ethnicity } \\
\hline Brahamin/Chettri & 1.00 (Reference) & 1.00 (Reference) & 1.00 (Reference) \\
\hline Terai/Madhesi other & $0.6(0.2-1.7)$ & $0.9(0.4-2.0)$ & $1.9 *(1.0-3.6)$ \\
\hline Dalits & $1.1(0.5-2.8)$ & $0.7(0.4-1.4)$ & $1.3(0.8-2.2)$ \\
\hline Newar/Janajatis & $0.6(0.3-1.2)$ & $0.6 *(0.4-0.9)$ & $1.1(0.8-1.5)$ \\
\hline Muslim/Other & $0.3(0.1-1.1)$ & $0.7(0.2-1.9)$ & $1.1(0.6-2.1)$ \\
\hline \multicolumn{4}{|l|}{ Place of delivery\# } \\
\hline Public facility & 1.00 (Reference) & 1.00 (Reference) & 1.00 (Reference) \\
\hline Private facility/NGOs & $1.5(0.9-2.8)$ & $3.2 * * *(2.2-4.6)$ & $3.6 * * *(2.7-4.9)$ \\
\hline Outside Nepal & NA & NA & $0.1^{* *}(0.0-2.5)$ \\
\hline
\end{tabular}

\#Home deliveries were excluded from the analysis.

Cesarean section was determined for the most recent birth of women aged 15-49 years who had a live-birth five years preceding the surveys. 
Each variable in the model has been adjusted by all the given variables in this table including age and parity.

NA: not available

$* * * \mathrm{p}<0.001, * * \mathrm{p}<0.01, * \mathrm{p}<0.05$

\section{Discussion}

In this study, we examined the trend of CS rate in Nepal and inequalities in its utilization by geography (Mountain, Hill and Terai); place of residence (urban/rural, Province); population characteristics (educational status, income quintile, caste/ethnicity, occupational status) and place of delivery. We found that overall CS rate in Nepal in 2016 (9\%) was below WHO's maximum limit of $15 \%$ and was much lower than in China $(43,44)$, Bangladesh (26)and Brazil(27). The current analysis revealed more than three-fold increase (from 3.2\%;95\% Cl: 2.1-4.3 in 2006 to $10.5 \%, 95 \%$ Cl: 8.9-11.9 in 2016) in age and parity adjusted CS rate over the decade. The rising trend in CS found in our study is consistent with the experience of other low and middle income countries $(45,46)$. This increase is largely due to increase in the proportion of institutional deliveries. Between 2006 and 2016, proportion of institutional deliveries (at public and private/NGO run facilities) in Nepal increased from $17.7 \%$ to $54 \%(14,28)$. Similarly, expansion of 24 hour birthing centers and emergency obstetric care in selected facilities has eased identification of possible complications and referral to higher facilities(5). Furthermore, in the fiscal year 2016/17, CS service was expanded to 72 districts(47), which was available only in 45 districts until 2009/10 (48) out of then 75 districts in Nepal. Additionally, GoN has implemented demand side financing scheme all over Nepal since 2009 to remove financial barriers to receive institutional deliveries, including CS when needed(5). While service expansion and demand side financing played a major role in increasing CS rate in Nepal, role of patients' preferences (choice of private facility over public facility, lower pain, choice of exact moment for delivery based on astrological belief) and provider preferences(save time, manage scheduling, increase earning, avoid litigation/ harassment)(45) cannot be overlooked. Moreover, increase in maternal age at first birth, growing proportion of pregnant women with the history of CS, and improvement in CS procedure may be associated with increase in CS rates as seen in other settings $(49,50)$.

Current analysis also revealed that while CS rate among the richest quintile increased (from $10.8 \%$ in 2006 to $25.1 \%$ in 2016) , it declined among women from the poorest income quintile after 2011 initially increasing from the level of 2006. Additionally, both the absolute and relative inequalities were larger by wealth quintile (rate difference: 22.7, rate ratio:10.5) compared to maternal educational status in 2016 (rate difference: 15.2, rate ratio:3.5). These findings clearly show that significant barriers exist for women from the poorest income quintiles

to access CS despite the existence of demand side financing scheme that cover transportation incentives and free delivery. A study by Acharya et al showed that average hidden cost (food and drinking, transportation, cost for childcare, cost for cloths) for delivering by CS in tertiary hospitals in Western Nepal was USD 321.6(51), and the cost was significantly higher to deliver at private hospital.

Furthermore, women with higher educational level (21.2\%;95\% Cl:14.7-27.8) had a higher CS rate than women with no education $(6.3 \% ; 95 \% \mathrm{Cl}: 3.5-9.0)$ after adjusting for maternal age and parity (Table 3 ). However, the association disappeared in multivariate regression analysis (Table 4). We previously found inequalities in full vaccination of children was larger by household wealth quintiles than by maternal educational status(52). Altogether, these findings suggest economic barriers for service utilization to be stronger than maternal educational related barriers in Nepal for maternal health and immunization service utilization. Between 2006 and 
2016, Rate Difference by maternal education increased while, Rate Ratio decreased from 8.5 in 2006 to 3.5 in 2016. Literature suggests that Rate Ratio decreases as the service coverage increase from overall lower level, whereas Rate Difference increases as the coverage reaches from low level to intermediate level (37).

Multivariate regression analysis also detected significant inequalities by provinces on the use of CS in comparison to Province 1. In addition to demand side factors (socio-cultural factors, wealth status, educational factors), unavailability/irregular functionality of birthing centers, and emergency obstetric services and geographical difficulties might have contributed to lower service utilization in some provinces and remote areas.

We found that women delivering in private facility $(37.1,95 \% \mathrm{Cl}: 30.5-43.7)$ have much higher CS rates than WHO's recommended level. Age and parity adjusted C-section rate in private facilities more than doubled from $15.8 \%$ in 2006 to $37.1 \%$ in 2016 while it remained almost constant $(12.3 \%$ in 2006 to $12.6 \%$ in 2016 ) in public facilities over ten years. In 2016, larger increase in proportion of deliveries in public facilities than in private facilities between 2006 and 2016 and constant CS rate in public facilities led to a slight overall increase in CS rate among deliveries conducted at HFs (Public+Private). In fact, women delivered in private/NGO-run facilities were nearly 4 times more likely to have CS (adjusted OR 3.6; 95\% Cl: 2.7-4.9) compared to women delivering in Public facilities. Such a high proportion of CS in these facilities suggest that non-medical factors (economic gain, manage scheduling or others) might have motivated health care providers to perform CS, especially in private HFs. The rise in C-section in Brazil especially among women of upper and middle class delivering in private sector was found to be induced by provider rather than by consumer preferences (4). The authors concluded that obstetricians perceived CS to be safer than vaginal delivery, or they might assume women in private facilities would prefer CS without exploring women's choices and expectations. Alternatively, it was suggested that given the staff constrains and workload issues, CS might be an easier option to manage schedules for managers and service providers(4). Constant CS rate in public facilities indicate relatively rational use of CS only when medically indicated.

\section{Implications}

On the basis of current findings and earlier findings by Acharya et al showing significant hidden cost to utilize CS (51) , the maternity incentive program needs to be revised targeting poor women from remote areas /mountain region areas when maternity complications arise. Although, GoN has established emergency referral fund to airlift women from poor, Dalit, Janajati, geographically disadvantaged and from mountainous region to transport to a higher facility in case of life threatening maternity complications, the use of this fund is very low. Only nine women in FY 2017/2018 used this fund to be air lifted(5). There is a need of an in-depth study to understand barriers in the use of emergency referral funds.

It is beyond the scope of this study to determine the cause for high C-section in private facilities. However, such a high CS rate in private/NGO-run facilities in Nepal warrants an appropriately defined quality assurance mechanism (53). Appropriate government policies and clinical practice protocols should be enacted to achieve an optimal C-section rates. Increasing rates of C-section has created a huge financial burden for Bangladesh's health care system (26). A study on an economic burden of CS in Nepal may be a starting point to understand it's significance. The findings can be used to develop advocacy tool, eventually to implement a contextual and validated tool such as Robson classification to support health workers to assess need and monitor the use of $\mathrm{CS}(54)$. Monitoring of the CS rate is necessary to ensure those who need the service are receiving and equity in health care use is achieved. Further, it can minimize provider induced CS for the sake of money. Additionally, 
ensuring continuous support (emotional support, information support, and advocacy of her wishes) for women during child-birth(55), and enhancing quality of obstetric care by ensuring adequate pain management, and use of technology can build-up women's confidence in obstetric care and decrease maternal demand for $\operatorname{CS}(23)$.

Some studies have indicated that midwife led maternity care can reduce $\mathrm{C}$-section rates including the maternity costs(56). In Nepal, maternity care in hospitals is obstetrician led and it is high time to consider promoting physiological birth through midwife led maternity care(24).There is a need to scale up current midwifery education to improve maternity care as well as to reduce over-burden of normal deliveries in tertiary care facilities.

Although the overall CS rates in Nepal is within WHO standard [15], due attention is necessary to monitor the increasing C-section rate (crude CS rate from 2.7\% in 2006 to $9 \%$ in 2016) and appropriate measures should be in place to curb the increasing trend. More importantly, women from the poorest income quintiles with maternity complications need special targeting. Furthermore, there is a need to expand C-section in Mountain districts and some provinces (Province 2, Province 5, Province 6 and Province 7) to reduce geographical inequality.

\section{Strengths and limitations}

We used high quality nationally representative data to assess trend and inequalities in CS. However, DHS survey lacks clinical data to evaluate appropriateness of CS. However, it is unlikely that clinical factors drive these variations. Furthermore, supply side variables and variables related to socio-cultural norms and beliefs were not available. Therefore, future studies can utilize supply side data from national surveys such as Nepal Health Facility Survey (57) for combining with DHS data to get a wider picture of the factors affecting CS utilization(58). Furthermore, qualitative studies are needed to understand service users' and providers' perspectives determining CS utilization.

\section{Conclusion}

In this paper, we found age and parity adjusted CS rate in Nepal to have increased almost three fold within ten years' period and is consistently high among women from the richest income quintiles, those with higher education and those delivering in private sector. This study also reveals that inequality persists and is increasing among some population groups (by maternal educational status, household wealth quintile) and provinces.

Strategies are needed to support mothers from the poor households, those from mountain region and those from province 2 , province 5 , province 6 and province 7 to utilize $C$-section when there is a medical need. Contextual and evidence- based clinical guidelines such as the Robson classification with frequent monitoring visits, especially in private facilities would help reduce unnecessary C-section.

\section{List Of Abbreviations}

$\mathrm{Cl} \quad$ Confidence Interval

CS Cesarean Section

C-section Cesarean Section

DHS Demographic and Health Survey 


$\begin{array}{ll}\text { GoN } & \text { Government of Nepal } \\ \text { NDHS } & \text { Nepal Demography and Health Survey } \\ \text { NGO } & \text { Non-governmental Organization } \\ \text { NHRC } & \text { Nepal Health Research Council } \\ \text { OR } & \text { Odds Ratio } \\ \text { SLC } & \text { School Leaving Certificate } \\ \text { USAID } & \text { United States Agency for International Development } \\ \text { WHO } & \text { World Health Organization }\end{array}$

\section{Declarations}

\section{Ethics approval and consent to participate}

All three round of DHS surveys in Nepal were approved by the Institutional Review Board of Nepal Health Research Council, Nepal; consent was sought before beginning the interview as per NHRC ethical review guidelines; data were publicly available and did not include individual identities and thus did not require ethics approval.

\section{Consent for publication}

Not Applicable

\section{Availability of data and materials}

Data is easily accessible from the DHS program website (https://dhsprogram.com/Data/terms-of-use.cfm) upon the request.

\section{Competing interests}

The authors declare that there are no conflicts of interest regarding the publication of this paper. The views and opinions expressed in this article are those of the authors and do not necessarily reflect the official policy or position of the organizations authors are affiliated with.

\section{Funding}

The authors received no specific grant from any funding agency in public, commercial, or not-for-profit sectors.

\section{Authors' contributions}


Conceptualization (YRP and KA), Data Analysis (KA), Methodology (YRP and KA), Supervision (YRP, KA and CMD), Validation (YRP, KA and CMD), Writing-original draft, review and editing (YRP, KA and CMD), read and approved the final version of the manuscript (YRP, KA and CMD)

\section{Acknowledgements}

The authors would like to thank the USAID's DHS program for providing the NDHS datasets.

\section{Author Information}

Kiran Acharya, Yuba Raj Paudel and Chandra Mani Dhungana

* Kiran Acharya and Yuba Raj Paudel contributed equally to this work

\section{Affiliations}

Kiran Acharya (Email-acharya.kiran1@gmail.com)

New ERA Nepal, Rudramati Marga, Kathmandu, Nepal

Yuba Raj Paudel (Email-yubaraj.paudel@gmail.com)

School of Public Health, University of Alberta, Edmonton, Canada

Chandra Mani Dhungana (Email- cmdhungana@gmail.com)

United Nations Population Fund, New York, USA

\section{Corresponding author}

Correspondence to Kiran Acharya

\section{References}

1. Ronsmans C, De Brouwere V, Dubourg D, Dieltiens G. Measuring the need for life-saving obstetric surgery in developing countries. BJOG: An International Journal of Obstetrics \& Gynaecology. 2004;111(10):1027-30.

2. Barros FCd, Victora C, Vaughan JP, Huttly S. Epidemic of caesarean sections in Brazil. The Lancet. 1991;338(8760):167-9.

3. Belizán JM, Showalter E, Castro A, Bastian H, Althabe F, Barros FC, et al. Rates and implications of caesarean sections in Latin America: ecological studyCommentary: all women should have a choiceCommentary: increase in caesarean sections may reflect medical control not women's choiceCommentary:"health has become secondary to a sexually attractive body". Bmj. 1999;319(7222):1397-402.

4. Potter JE, Berquó E, Perpétuo IH, Leal OF, Hopkins K, Souza MR, et al. Unwanted caesarean sections among public and private patients in Brazil: prospective study. Bmj. 2001;323(7322):1155-8.

5. DoHS. Annual Report. Kathmandhu, Nepal: Ministry of Health and Population, Department of Health Services (DoHS); 2017/2018. 
6. Ministry of Health Nepal. Nepal Health Sector Strategy 2015-2020: Ministry of Health, Nepal; 2015. Available from: http://nhsp.org.np/nepal-health-sector-strategy-2015-2020/.

7. National Planning Commission. Sustainable Development Goals, 2016-2030, National (Preliminary) Report. . Kathmandu: Government of Nepal, National Planning Commission; 2015.

8. Tiwari Suresh, Bhatt Hema, EnsorTimothy , Suvedi BK, Lievens Tomas, James Chris, et al. Unit cost analysis of the health facility reimbursement made under the Aama Programme. Kathmandu, Nepal: Ministry of Health and Population/Family Health Division and Nepal Health Sector Support Programme.; 2015.

9. Family Health Division. Aama Circular 20752018: Available from: http://fhd.gov.np/images/notice/Aama_Circular_2075.pdf.

10. Family Health Division DoHS, Ministry of Health and Population,. Aama Programme, Implementation Guidelines, Second Amendment 2009. Kathmandu, Nepal 2012.

11. Subedi S, Kaphle HP, Acharya S, Gupta N, Jain V. Maternal incentive scheme in Nepal-status of knowledge and financial benefits received by mothers on ANC incentive and safe delivery incentive program. Indian Journal of Community Health. 2014;26(3):273-7.

12. Witter S, Khadka S, Nath H, Tiwari S. The national free delivery policy in Nepal: early evidence of its effects on health facilities. Health policy and planning. 2011;26(suppl_2):ii84-ii91.

13. Pradhan A. Nepal family health survey, 1996: Family Health Division, Dept. of Health Services, Ministry of Health; 1997.

14. Ministry of Health and Population (MoHP) Nepal, New ERA, and ICF International Inc. Nepal Demographic and Health Survey 2016. Kathmandu: Ministry of Health and Population2017.

15. Bhatt H, Tiwari S, Ensor T, Ghimire DR, Gavidia T. Contribution of Nepal's free delivery care policies in improving utilisation of maternal health services. International journal of health policy and management. 2018;7(7):645.

16. Khanal V, Karkee R, Lee AH, Binns CW. Adverse obstetric symptoms and rural-urban difference in cesarean delivery in Rupandehi district, Western Nepal: a cohort study. Reproductive Health. 2016 2016/03/01;13(1):17.

17. Mehata S, Paudel YR, Dariang M, Aryal KK, Lal BK, Khanal MN, et al. Trends and inequalities in use of maternal health care services in Nepal: strategy in the search for improvements. BioMed research international. 2017;2017.

18. Ronsmans C, Graham WJ, group LMSSs. Maternal mortality: who, when, where, and why. The Lancet. 2006;368(9542):1189-200.

19. Jahnke JR, Houck KM, Bentley ME, Thompson AL. Rising rates of cesarean delivery in Ecuador: Socioeconomic and institutional determinants over two decades. Birth. 2019.

20. Vishwakarma R, Verma V, Nath DC, Prakash R, Abid O. Prevalence and Determinants of Caesarean Section in South and South-east Asian Women. BioRxiv. 2019:616813.

21. Family Health Division and Nepal Health Sector Support Programme. Rapid Assessment of the Demand Side Financing Scheme: Aama Surakshya Programme (The Ninth Rapid Assessment) 2016. Available from: http://www.nhssp.org.np/NHSSP_Archives/health_financing/Aama_Rapid_Assessment_IX_August_2016.pdf.

22. Gamble J, Creedy DK, McCourt C, Weaver J, Beake S. A critique of the literature on women's request for cesarean section. Birth. 2007;34(4):331-40. 
23. Schantz C, de Loenzien M, Goyet S, Ravit M, Dancoisne A, Dumont A. How is women's demand for caesarean section measured? A systematic literature review. PloS one. 2019;14(3).

24. Dhakal Rai S, Regmi P, Van Teijlingen E, Wood J, Dangal G, Dhakal K. Rising Rate of Caesarean Section in Urban Nepal. Journal of Nepal Health Research Council. 2019;16(41):479-80.

25. Betrán AP, Torloni MR, Zhang J-J, Gülmezoglu A, Section WWGoC, Aleem H, et al. WHO statement on caesarean section rates. BJOG: An International Journal of Obstetrics \& Gynaecology. 2016;123(5):667-70.

26. Haider MR, Rahman MM, Moinuddin M, Rahman AE, Ahmed S, Khan MM. Ever-increasing Caesarean section and its economic burden in Bangladesh. PloS one. 2018;13(12):e0208623.

27. Freitas PF, Drachler MdL, Leite JCdC, Marshall T. Inequalities in cesarean delivery rates by ethnicity and hospital accessibility in Brazil. International Journal of Gynecology \& Obstetrics. 2009;107(3):198-201.

28. Ministry of Health and Population (MoHP) Nepal, New ERA, and ICF International Inc. Nepal Demographic and Health Survey 2011. Kathmandu, Nepal 2012.

29. Ministry of Health and Population (MoHP) Nepal, New ERA, and ICF International Inc. Nepal Demographic and Health Survey 2006. Kathmandu: Ministry of Health and Population2007.

30. Collin SM, Anwar I, Ronsmans C. A decade of inequality in maternity care: antenatal care, professional attendance at delivery, and caesarean section in Bangladesh (1991-2004). International journal for equity in health. 2007;6(1):9.

31. Mumtaz S, Bahk J, Khang Y-H. Rising trends and inequalities in cesarean section rates in Pakistan: Evidence from Pakistan Demographic and Health Surveys, 1990-2013. PloS one. 2017;12(10).

32. Soto-Vega E, Casco S, Chamizo K, Flores Hernández D, Landini V, Guillén-Florez A. Rising trends of cesarean section worldwide: a systematic review. Obstet Gynecol Int J. 2015;3(2):00073.

33. Rutstein SO, Johnson K. The DHS wealth index. DHS comparative reports no. 62004.

34. Mackenbach JP, Kunst AE. Measuring the magnitude of socio-economic inequalities in health: an overview of available measures illustrated with two examples from Europe. Social science \& medicine. 1997;44(6):75771.

35. Paudel D, Shrestha IB, Siebeck M, Rehfuess EA. Neonatal health in Nepal: analysis of absolute and relative inequalities and impact of current efforts to reduce neonatal mortality. BMC Public Health. 2013;13(1):1239.

36. Anand S, Diderichsen F, Evans T, Shkolnikov VM, Wirth M. Measuring disparities in health: methods and indicators. Challenging inequities in health: from ethics to action. 2001:49-67.

37. Houweling TA, Kunst AE, Huisman M, Mackenbach JP. Using relative and absolute measures for monitoring health inequalities: experiences from cross-national analyses on maternal and child health. International journal for equity in health. 2007;6(1):15.

38. Moser K, Frost C, Leon DA. Comparing health inequalities across time and place-rate ratios and rate differences lead to different conclusions: analysis of cross-sectional data from 22 countries 1991-2001. International journal of epidemiology. 2007;36(6):1285-91.

39. King NB, Harper S, Young ME. Use of relative and absolute effect measures in reporting health inequalities: structured review. Bmj. 2012;345:e5774.

40. Mackenbach JP, Kunst AE. Measuring the magnitude of socio-economic inequalities in health: an overview of available measures illustrated with two examples from Europe. 1997. 
41. Mahaini R, Gholbzouri K, Jadoon B. Determinants of over and underuse of caesarean births in the Eastern Mediterranean Region: an updated review. East Mediterr Health J. 2019.

42. World Health Organization. Appropriate technology for birth. Lancet. 1985;2:436-7.

43. Mi J, Liu F. Rate of caesarean section is alarming in China. The Lancet. 2014;383(9927):1463-4.

44. Li H-T, Luo S, Trasande L, Hellerstein S, Kang C, Li J-X, et al. Geographic variations and temporal trends in cesarean delivery rates in China, 2008-2014. JAMA. 2017;317(1):69-76.

45. Sharma G. The changing paradigm of labour and childbirth in Indian cities: an enquiry into increasing rates of caesarean deliveries. International Journal of Epidemiology. 2016;45(5):1390-3.

46. Mumtaz S, Bahk J, Khang Y-H. Rising trends and inequalities in cesarean section rates in Pakistan: Evidence from Pakistan Demographic and Health Surveys, 1990-2013. PloS one. 2017;12(10):e0186563.

47. Ministry of Health Nepal. Annual Report, Department of Health Services 2073/2074 (2016/2017). Ministry of Health, Nepal; 2018.

48. Ministry of Health Nepal. Annual Report, Department of Health Services 2066/2067(2009/2010). Ministry of Health, Nepal; 2011.

49. Porreco RP, Thorp JA. The cesarean birth epidemic: trends, causes, and solutions. American journal of obstetrics and gynecology. 1996;175(2):369-74.

50. Rajabi A, Maharlouei N, Rezaianzadeh A, Rajaeefard A, Gholami A. Risk factors for C-section delivery and population attributable risk for C-section risk factors in Southwest of Iran: a prospective cohort study. Medical journal of the Islamic Republic of Iran. 2015;29:294.

51. Acharya J, Kaehler N, Marahatta SB, Mishra SR, Subedi S, Adhikari B. Hidden costs of hospital based delivery from two tertiary hospitals in western Nepal. PloS one. 2016;11(6).

52. Acharya K, Paudel YR, Dharel D. The trend of full vaccination coverage in infants and inequalities by wealth quintile and maternal education: analysis from four recent demographic and health surveys in Nepal. BMC Public Health. 2019;19(1):1673.

53. Robson M, Hartigan L, Murphy M. Methods of achieving and maintaining an appropriate caesarean section rate. Best practice \& research Clinical obstetrics \& gynaecology. 2013;27(2):297-308.

54. Betran AP, Vindevoghel N, Souza JP, Gülmezoglu AM, Torloni MR. A systematic review of the Robson classification for caesarean section: what works, doesn't work and how to improve it. PloS one. 2014;9(6):e97769.

55. Hodnett ED, Gates S, Hofmeyr GJ, Sakala C. Continuous support for women during childbirth. Cochrane database of systematic reviews. 2013(7).

56. Donahue S, Sunday M. Effect of Midwife Led Care on Cesarean Section Rate. 2015.

57. Ministry of Health Nepal, New ERA Nepal, Nepal Health Sector Support Program (NHSSP), and ICF. Nepal Health Facility Survey 2015.: Ministry of Health, Nepal; 2017.

58. Wang W. Influence of service readiness on use of facility delivery care: a study linking health facility data and population data in Haiti: ICF International; 2014. 